Pacific Journal of Mathematics

HIGH SUBGROUPS OF ABELIAN TORSION GROUPS 


\section{HIGH SUBGROUPS OF ABELIAN TORSION GROUPS}

JOHN M. IRWIN

The results in this paper were part of a doctor's thesis completed in February 1960 under Professor W. R. Scott at the University of Kansas. The author wishes to express his gratitude to Professor Scott for his advice and for checking the results.

In what follows, all groups considered are Abelian. Let $G^{1}$ be the subgroup of elements of infinite height in an Abelian group $G$ (see [2]). A subgroup $H$ of $G$ maximal with respect to disjointness from $G^{1}$ will be called a high subgroup of $G$. If $N$ is a subgroup of $G, H$ will be called $N$-high if and only if $H$ is a subgroup of $G$ maximal with respect to disjointness from $N$. Zorn's lemma guarantees the existence of $N$ high subgroups for any subgroup $N$ of $G$. A group $E$ minimal divisible among those groups containing $G$ will be called a divisible hull of $G$. Unless otherwise specified, the notation and terminology will be essentially that of L. Fuchs in [1].

The main theorem says that high subgroups of Abelian torsion groups are pure. After proving some preparatory lemmas, we will prove the main theorem. Then we will discuss Fuchs' Problem 4 and list some of the more important properties of high subgroups. Finally we will state some generalizations.

A lemma describing $N$-high subgroups is

LemMA 1. Let $G$ be a primary group with $H$ an $N$-high subgroup of $G, D$ a divisible hull of $G, A$ any divisible hull of $H$ in $D$ (this means that $A \subset D$ ), and $B$ any divisible hull of $N$ in $D$.

Then

(a) $D=A \oplus B$.

(b) $A \cap G=H$, and $H$ and $B \cap G$ are neat in $G$.

(c) Any complementary direct summand of $A$ in $D$ containing $N$ is a divisible hull of $N$.

(d) Any complementary direct summand of $B$ in $D$ containing $H$ is a divisible hull of $H$.

(e) $D$ is a divisible hull of any subgroup $M$ with $(H \oplus N)[p] \subset$ $M \subset G$.

(f) $D[p]=(H \oplus N)[p]=H[p] \oplus N[p]=G[p]$.

(g) All $N$-high subgroups $H$ of $G$ may be obtained as $E \cap G$, where $E$ is a complementary direct summand of a divisible hull $F$ of $N$ in $D$.

Proof. When $N=0$ there is nothing to prove, so suppose $N \neq 0$.

Received October 31, 1960. 
(a) To see this, we first show that $A \cap B=0$. If $0 \neq x \in A \cap B$, then by Kulikov's lemma ([1], p.66) there exist positive integers $r$ and $s$ such that $p^{r} x \neq 0 \neq p^{s} x$ and $p^{r} x \in H, p^{s} x \in N$. But $0 \neq p^{\max (r, s)} x \in H \cap N$ $=0$, which is impossible. The divisibility of $A \oplus B$ provides a decomposition $D=A \oplus B \oplus C$. If $C \cap G \neq 0, H$ will not be $N$-high in $G$, whence $C \cap G=0$. By Kulikov's lemma, $C=0$, and we have $D=A \oplus B$ as stated.

(b) That $A \cap G=H$ is clear. The neatness follows from [1], p. 92, $h$,

(c) and (d) follow from (a) and the definition of $H$,

(e) and (f) follow from Kulikov's lemma,

(g) follows from (a) and (b). This concludes the proof of Lemma 1.

In what follows $\langle x\rangle$ will denote the cyclic subgroup of $G$ generated by $x \in G$. An interesting and helpful lemma is

Lemma 2. Let $N$ be a subgroup of a primary group $G, H$ an $N$ high subgroup of $G$, and let $H$ contain a basic subgroup $B$ of $G$. Then $H$ is pure in $G$.

Proof. The group $G / B$ is divisible since $B$ is basic in $G$. Now $H / B \subset G / B$, and by [1], p. 66, Theorem 20.2, there exists a divisible hull $E / B$ of $H / B$ in $G / B$. Suppose $E / B>H / B$. Then $E>H$, and hence $E \cap N \neq 0$. Thus there exists a nonzero element $g \in N$ with $0 \neq$ $\langle g+B\rangle \subset E / B$. Now $\langle g+B\rangle \cap(H / B)=0$. To see this, suppose $0 \neq$ $m(g+B)=m g+B=h+B$. Then $m g-h=b \in B$, and $0 \neq m g=$ $h+b \in H$, contradicting $H \cap N=0$. Thus we have $\langle g+B\rangle \cap(H \mid B)=$ 0 . By Kulikov's lemma, $\langle g+B\rangle=0$, and therefore $g \in B$, which implies that $g=0$, contrary to the choice of $g$. Thus $E / B=H / B$ is divisible, and therefore is pure in $G / B$. Then the purity of $B$ in $G$ together with [1], p. 78, $M$ imply that $H$ is pure in $G$.

A useful lemma with a standard proof is

Lemma 3. If $G=S \oplus T$, where each element of $S$ has finite height, then $G^{1} \subset T$ and $T^{1}=G^{1}$.

A lemma which displays an inheritance property is

Lemma 4. If $G=S \oplus T$, where $S \subset H$ and $H$ is high in $G$, then $H=S \oplus H \cap T$, and $H \cap T$ is high in T. (Note: This implies that $H \cap T$ is maximal with respect to disjointness from $G^{1}$ in $T$ by Lemma 3.)

Proof. Put $M=H \cap T$, and suppose that there exists $0 \neq t \in T \backslash H$, with $\{M, t\} \cap T^{1}=0$. But this means that $\{M, t\} \cap G^{1}=0$, and hence $[S \oplus\{M, t\}] \cap G^{1}=0$; for otherwise we would have $s+(m+k t)=g \neq 0$ with $s \in S, m \in M, g \in G^{1}$. Then $s=0$ and $m+k t=g \neq 0$. But $m+$ $k t \in\{M, t\}$, which is not possible. Thus $[S \oplus\{M, t\}] \cap G^{1}=0$ and 
$[S \oplus\{M, t\}]>H$, contrary to the assumption that $H$ is high in $G$.

A lemma on making new basic subgroups out of old ones is

Lemma 5. Let $B_{1} \oplus \cdots \oplus B_{n} \oplus G_{n}=G$, where $B=\Sigma B_{n}$ is basic in G. Let $T_{n}=B_{n+1}^{\prime} \oplus B_{n+2}^{\prime} \oplus \cdots$ be basic in $G_{n}$. Then $C=B_{1} \oplus \cdots \oplus$ $B_{n} \oplus T_{n}$ is basic in $G$.

\section{Proof [1], p. 109, Exercise 9a.}

An $x \in G$ will be called a pure element of $G$ if and only if $\langle x\rangle$ is a pure subgroup (and therefore is a direct summand) of $G$.

The next lemma is the kingpin in the proof that if $H$ is high in $G$, then $H$ contains $B$ basic in $G$. It is not altogether obvious that $H$ contains nonzero pure subgroups of $G$. The proof of the next lemma will be carried out in several steps. We will consider special cases which are perhaps unnecessary, but which will help to clarify the method of proof.

LEMMA 6. Let $H$ be a high subgroup of a primary group $G$. If $G$ contains nonzero pure elements of order $p^{n}$, but not of smaller order, then $H$ contains pure elements of $G$ of order $p^{n}$.

\section{Proof.}

Case 1. $n=1$. Let $b \in G$ be pure of order $p$ with $b \notin H$. Then there exists $h \in H$ such that $h+b=g \neq 0$, where $g \in G^{1}$. Clearly this means that the orders of $h$ and $b$ are the same. Now $h$ and $b$ both have finite height, and hence their heights must be equal (since their sum is an element of infinite height in $G$ ). Here we are making use of the fact that if $o(h)=p$ and $h(h)=0$, then $\langle h\rangle$ is pure in $G$. The fact that $b$ is a pure element of order $p$ in $G$ necessarily means that $h(b)=0$; whence $h(h)=0$, and $h$ is a pure element of $G$.

Case 2. $n>1$. Let $b$ be a pure element of $G$ of order $p^{n}$ such that $b \notin H$. Then there exists an $h \in H$ such that $h+p^{j} b=g \neq 0$, where $g \in G^{1}$ and $0 \leqq j<n$.

Case 2.1. $j=0$. Then we have $h+b=g$ and $p^{n-1} h+p^{n-1} b=$ $p^{n-1} g \in G^{1}$. Clearly $p^{n-1} h$ has order $p$ and height $n-1$ in $G$ and in $\langle h\rangle$. Thus by [1], p. 78, $J$, we have that $h$ is a pure element of $G$.

Case 2.2. $1 \leqq j<n$. Now the equation $h+p^{y} b=g \neq 0$ clearly implies that the height of $h$ in $G$ is $j$. If the height of $h$ in $H$ were also $j$, that is if $h=p^{j} h^{\prime}$ for some $h^{\prime} \in H$, then $h^{\prime}$ would be a pure 
element of $G$ of order $p^{n}$. To see this simply consider the equation $p^{n-1} h^{\prime}+p^{n-1} b=p^{n-1-1} g \in G^{1} . \quad o\left(p^{n-1} h^{\prime}\right)=p$ and obviously $h\left(p^{n-1} h^{\prime}\right)=$ $n-1$. The height of $p^{n-1} h^{\prime}$ in $\left\langle h^{\prime}\right\rangle=n-1$, so that by [1], p. 78, $J$, $h^{\prime}$ is a pure element of $G$ of order $p^{n}$ in $H$. Thus it remains to verify that the height of $h$ in $H$ is $j$.

From the neatness of $H$ and the fact that $g \in G^{1}$, it follows that $h=p h_{1}$ for some $h_{1} \in H$. Now if $h\left(h_{1}\right)>0$, we again have by the neatness of $H$ that $h_{1}=p h_{2}$ for some $h_{2} \in H$. Continuing in this way, we must eventually arrive at $h=p^{j-k} h_{k}, h_{k} \in H$ where the height of $h_{k}$ in $G$ is 0 . If $k>0$, then let $m$ be the least positive integer such that $p^{m} h_{k}=p^{m+1} z$ for $z \in G$ (if worst comes to worst $m=j-k$ will do). Then clearly $0<m \leqq j-k<j<n, \quad p^{m}\left(h_{k}-p z\right)=0$, and $p^{m-1}\left(h_{k}-p z\right) \neq 0$ has height $m-1$ in $G$ by the choice of $m$ and $h_{k}$. Thus since $o\left(p^{m-1}\left(h_{k}-p z\right)\right)$ $=p$, and the height of $p^{m-1}\left(h_{k}-p z\right)$ is $m-1$ in $\left\langle h_{k}-p z\right\rangle$, we have by [1], p. 78, $J$, that $h_{k}-p z$ is a pure element of $G$ of order $p^{m}<p^{n}$. This contradicts the hypotheses on $G$. Hence we must have $k=0$, $h=p^{j} h_{k}$, and $h_{k}$ is a pure element of $G$ in $H$ of order $p^{n}$.

If $B=\Sigma B_{n}$ is a basic subgroup of $G$ where $B_{n}$ is a direct sum of cyclic groups of order $p^{n}$, then such a subgroup $B_{n}$ which does not consist of 0 alone will be referred to as a $B_{n}$ of $G$.

Lemma 7. Let $G$ be a primary group, $H$ a high subgroup of $G$, and $n$ the least positive integer such that $G$ contains $a B_{n}$. Then $H$ contains $a B_{n}$ of $G$.

Proof. By Lemma 6, $H$ contains pure elements of $G$ of order $p^{n}$. The fact that the union of an ascending chain of pure subgroups is pure together with [1], p. 80, Theorem 24.5 allows us to apply Zorn's lemma to obtain a $p^{n}$-bounded direct summand $H_{n}$ of $G$, maximal with respect to the property of being contained in $H$. We wish to show that $H_{n}$ is a $B_{n}$ for $G$. To see this write $G=H_{n} \oplus R_{n}$ and $H=H_{n} \oplus H \cap R_{n}$ where by Lemma $4, H \cap R_{n}$ is high in $R_{n}$. Suppose that $H_{n}$ is not a maximal $p^{n}$-bounded direct summand $\left(\mathrm{a} B_{n}\right.$ ) of $G$. Then there exists a $B_{n}$ of $G$ with $H_{n}<B_{n}$. Now $G=H_{n} \oplus R_{n}$, so that $B_{n}=H_{n} \oplus B_{n} \cap R_{n}$. Now the transitivity of purity tells us that $B_{n} \cap R_{n} \neq 0$ is pure in $G$. Thus $R_{n}$ contains pure elements of order $p^{n}$ since $G$ contains no pure elements of order less than $p^{n}$. This means by Lemma 6 that $H \cap R_{n}$ as a high subgroup of $R_{n}$ must contain a pure element $h$ of order $p^{n}$. Then $G=H_{n} \oplus R_{n}=H \oplus\langle h\rangle \oplus R_{n}^{\prime}$, and $\left.\left(H_{n} \oplus\langle h\rangle\right)\right\rangle H_{n}$ implies that $H_{n}$ is not a maximal $p^{n}$-bounded direct summand of $G$ contained in $H$, contrary to the choice of $H_{n}$. This means that $H_{n}$ is a $B_{n}$ of $G$ contained in $H$ after all, and this concludes the proof.

LEMma 8. Let $G$ be a primary group, and let $H$ be a high sub- 
group of $G$. Then $H$ contains a basic subgroup of $G$.

Proof. By a theorem of Baer ([1], p.62), it suffices to consider the reduced case. Lemma 7 provides a start for the induction. Let $B_{n_{1}}^{\prime}$ be a first $B_{n}$ of $G$. By lemma $7, H$ contains a $B_{n_{1}}$ and $G=B_{n_{1}} \oplus R_{1}$ with $H=B_{n_{1}} \oplus H \cap R_{1}$. Let $B_{n_{2}}$ be the next $B_{j}$ of $G$. By Szele's theorem ([1], p. 99) and Lemma $5, R_{1}$ contains a $B_{n_{2}}$ but no preceding $B_{j}$. We apply Lemma 7 to $H \cap R_{1}$ as a high subgroup of $R_{1}$ to see that $H \cap R_{1}$ contains a $B_{n_{2}}$ of $G$. By successive application of this procedure, we have by induction, Szele's theorem, Lemmas 5 and 7 that $H$ contains a basic subgroup of $G$.

We are now ready to state and prove our main theorem.

THEOREM 1. Let $G$ be a primary group and $H$ a high subgroup of $G$. Then $H$ is pure in $G$.

Proof. As in the proof of Lemma 8, it suffices to consider the case where $G$ is reduced. Lemmas 2 and 8 complete the proof.

In his book [1], L. Fuchs asks the following question: "Let $G$ be a primary group and $H$ an infinite subgroup without elements of infinite height. Under what conditions can $H$ be imbedded in a pure subgroup of the same power and again without elements of infinite height?" Theorem 1 allows us to give the best possible solution to this problem.

Theorem 2. Let $G$ be an Abelian primary group. If $S$ is any infinite subgroup of $G$ with $S \cap G^{1}=0$, then $S$ can be embedded in a pure subgroup $K$ of $G$ so that $K \cap G^{1}=0$ and $|K|=|S|$.

Proof. By Zorn's lemma, there exists a subgroup $H$ high in $G$ with $H \supset S$. By Theorem 1, $H$ is pure in $G$. Szele has shown that every infinite subgroup can be embedded in a pure subgroup of the same power ([1], p. 78). So let $K$ be a pure subgroup of $H$ containing $S$ and of the same power as $S$. Then by the transitivity of purity, we have that $K$ is pure in $G$. Since $K \subset H$, it follows that $K \cap G^{1}=0$. This concludes the proof.

The following discussion yields the solution to Fuchs' question in the torsion case. The proofs of the next two lemmas are standard and consequently will be omitted.

Lemma 9. Let $G$ be a torsion group. If $G=\Sigma G_{\alpha}$, then $G^{1}=\Sigma G_{\alpha}^{1}$.

Lemma 10. Let $G$ be a torsion group. Then an internal direct sum of pure subgroups of the direct summands of a given direct decomposition of $G$ is a pure subgroup of $G$.

Concerning the primary decomposition of a torsion group $G$, we have, 
Lemma 11. If $H$ is a high subgroup of a torsion group $G$, then writing $G$ and $H$ in terms of their primary components $G=\Sigma G_{p}$ and $H=\Sigma H_{p}=\Sigma H \cap G_{p}$, we have that $H_{p}$ is a high subgroup of $G_{p}$ for each relevant prime $p$ in the primary decomposition of $G$.

Proof. Clearly $H_{p} \cap G_{p}^{1}=0$. So suppose for some $p, H_{p}$ is not high in $G_{p}$. Then there exists an $x \in G_{p} \backslash H_{p}$ with $\left\{H_{p}, x\right\} \cap G_{p}^{1}=0$. Replacing $H_{p}$ by $S_{p}=\left\{H_{p}, x\right\}$ in $H=\Sigma H_{p}$, we obtain from Lemma 9 a subgroup $S>H$ with $S \cap G^{1}=0$. But this is contrary to $H$ high in $G$.

\section{A generalization of Theorem 1 is}

THEOREM 3. If $H$ is a high subgroup of a torsion group $G$, then $H$ is pure in $G$.

Proof. Write $G=\Sigma G_{p}$ and $H=\Sigma H_{p}$ and by Lemma 11, we have that $H_{p}$ is high in $G_{p}$ so that by Theorem 1 we have $H_{p}$ is pure in $G_{p}$. Now by Lemma $10, H$ is pure in $G$.

The generalization of the solution to Fuchs' question to torsion groups is

THeORem 4. Any infinite subgroup $S$ of a torsion group $G$ with $S \cap G^{1}=0$ can be embedded in a pure subgroup $K$ of $G$ so that $|\boldsymbol{K}|=$ $|S|$ and $K \cap G^{1}=0$.

Proof. Use Theorem 3 and the proof of Theorem 2.

We mention for completeness that Lemma 8 has a suitable generalization to torsion groups.

Lemma. 12. Let $G$ be a torsion group and let $H$ be a high subgroup of $G$. Then $H$ contains a basic subgroup of $G$.

Proof. Use Lemma 8, the primary decomposition of $H$, and [1], p. 109, Exercise 9a.

Some of the more interesting properties of high subgroups are contained in

THEOREM 5. Let $G$ be a reduced primary group with $G^{1} \neq 0$, and let $H$ and $K$ be high subgroups of $G$. Then

(a) $H$ contains $B$ basic in $G$

(b) $H$ is pure in $G$

(c) $G / H$ is a divisible hull of $\left(G^{1} \oplus H\right) / H \cong G^{1}$

(d) $G \mid K \cong G / H$

(e) $p^{n} H$ is high in $p^{n} G$ for all $n \in I$ ( $I$ is the set of positive integers.) 
(f) $p^{n} H$ is pure in $p^{n} G$ for all $n \in I$

(g) $G=\left\{H, p^{n} G\right\}$ for all $n \in I$

(h) $H$ is infinite

(i) $H$ is of unbounded height in $G$

(j) $p^{n} G=\left\{p^{n} H, p^{n+k} G\right\}$ for all $n, k \in I$

(k) $p^{n} H / p^{n+k} H \cong p^{n} G / p^{n+k} G$ for all $n, k \in I$.

(l) $p^{n} G / p^{n} H \cong G / H$ for all $n \in I$.

(m) $G$ is minimal pure containing $H \oplus G^{1}$

(n) $H \oplus G^{1}<G$

(o) $\left|H \oplus G^{1}\right|=|G|$

(p) $\left|G^{1}\right|<|G|$ implies $|H|=|G|$

(q) $|K|=|H|$ (This also holds for $N$-high subgroups of infinite rank.)

(r) $|G| \leqq|H|^{\aleph_{0}}$

(s) $\quad G / p^{n} H=H / p^{n} H \oplus p^{n} G / p^{n} H$ for all $n \in I$.

(t) $H$ is not always basic

(u) If $H$ is countable, then $H$ is basic in $G$, and $H \cong K$.

Proof. (a) and (b) have already been proved.

(c) Is easy.

(d) Follows from (c) and the fact that isomorphic groups have isomorphic divisible hulls (see [1], p. 66, Theorem 20.2).

(e) To see that $p^{n} H$ is high in $p^{n} G$, suppose that there exists $x \in G$ with $\left\{p^{n} H, p^{n} x\right\} \cap G^{1}=0$ and $p^{n} x \notin p^{n} H$. (Here we are using the fact that $\left(p^{n} G\right)^{1}=G^{1}$.). Now by purity of $H, p^{n} x \notin p^{n} H$ implies $p^{n} x \notin H$. Thus we have some $h \in H$ with $h+m p^{n} x=g \neq 0, g \in G^{1}$. But then $h$ must be in $p^{n} H$ contrary to $\left\{p^{n} H, p^{n} x\right\} \cap G^{1}=0$.

(f) The purity of $p^{n} H$ in $p^{n} G$ follows from (e), and Theorem 1 applied to $p^{n} G$.

(g) This is an immedite consequence of (c).

(h) And (i) both follow from (g) and the fact that a high subgroup of a reduced group is not a direct summand.

(j) Follows from (e) and (g).

(k) Follows from $(j)$, the second isomorphism theorem, and ( $f$ ).

(l) Is an immediate consequence of the fact that both quotient groups are divisible hulls of $G^{1}$. This is also a straightforward application of $(\mathrm{g})$.

(m) This follows from Lemma 1 (f) and [1], p. 78, $K$.

(n) Follows from the fact that (c) holds and hence $H$ is not a direct summand of $G$.

(o) Follows from Lemma 1 (f) and an easy set theoretic argument.

(p) Is an easy consequence of (o).

(q) Here some cases are taken care of by (d), but a proof for the general case is not difficult. To show that $|H|=|K|$, it suffices (by an 
easy set theoretic argument) to verify that $H[p] \cong K[p]$. For this purpose let $D$ be a divisible hull of $G$, and $C$ be a divisible hull of $G^{1}$ in $D$. By Lemma 1 , if $A$ and $B$ are divisible hulls in $D$ of $H$ and $K$ respectively, then $A$ and $B$ are complementary direct summands of $C$ in $D$. Finally $A \cong D / C \cong B$ and $H[p]=A[p] \cong B[p]=K[p]$. The same argument shows the result for $N$-high subgroups of infinite rank.

(r) Follows trivially from (a) and [1], p. 102, Theorem 30.1.

(s) To see this, use (g) and the purity of $H$.

(t) Let $G$ be the direct sum of an unbounded closed primary group and any primary group with nonzero elements of infinite height.

(u) This follows from (b), (c), (q), the fact that a countable $H$ is a direct sum of cyclic groups, and that any two basic subgroups of $G$ are isomorphic.

For a comparison with the properties of basic subgroups see [1], p. 101. The reader will notice that (d) is an interesting property of high subgroups which basic subgroups do not possess.

We are now ready to discuss the question of whether or not any two high subgroups of a reduced primary group are isomorphic. Let $A$ be a subgroup of $G$, and let $\widetilde{A}$ be the image under the natural homomorphism from $G$ onto $G / G^{1}$. It is a simple matter to verify that $\widetilde{G}$ is a reduced primary group without elements of infinite height. Thus if $H$ is a high subgroup of $G$, we have that $H \cong \widetilde{H}$. This provides us a natural way to study the properties of high subgroups without actually looking at these subgroups themselves.

A result concerning Ulm invariants as defined by Kaplansky in [2], and providing another proof that two countable high subgroups of a group $G$ are isomorphic is the following

Theorem 6. Let $H$ and $K$ be high subgroups of a primary group G. Then $\left(p^{n} \widetilde{H}\right)[p] /\left(p^{n+1} \widetilde{H}\right)[p]=\left(p^{n} \widetilde{K}\right)[p] /\left(p^{n+1} \widetilde{K}\right)[p]$. In particular, $H$ and $K$ have the same Ulm invariants. Moreover, their nth Ulm invariants are the same as the nth Ulm invariant of $G$.

Proof. Consider $\tilde{H}$ and $\widetilde{K}$. First we notice

(i) $\widetilde{H}[p]=\widetilde{K}[p]$.

To see this we observe that $o(h)=o\left(h+G^{1}\right)$. Suppose $h \in H[p] \backslash H \cap$ $K$. Then there exists $k \in K$ with $h-k=g \neq 0$ where $g \in G^{1}$. Clearly $o(k)=p$ and we have $h=k+g$. This proves that $\widetilde{H}[p] \subset \widetilde{K}[p]$. Thus by symmetery $\widetilde{H}[p]=\widetilde{K}[p]$. Next we have

(ii) $p^{n} \widetilde{H}[p]=p^{n} \widetilde{K}[p]$ for $n \in I$.

To see this use Theorem 5 (e), and the foregoing (i).

Now from (ii) we have that $\left(p^{n} \widetilde{H}\right)[p] \backslash\left(p^{n+1} \widetilde{H}\right)[p]=\left(p^{n} \widetilde{K}\right)[p] /\left(p^{n+1} \widetilde{K}\right)[p]$ since the numerators are equal and the denominators are equal, and hence the Ulm invariants of $\widetilde{H}$ and $\widetilde{K}$ are equal. Finally the fact that 
$H \cong \widetilde{H}$ gives us that $H$ and $K$ have the same $n$th Ulm invariants. The last part of the theorem follows from $\left(p^{n} G\right)[\mathrm{p}] /\left(p^{m+1} G\right)[p] \cong\left(p^{n} \tilde{H}\right)[p] /\left(p^{n+1} \widetilde{H}\right)[p]$ which is obtained with the help of Lemma $1(\mathrm{f})$, Theorem $5(\mathrm{e})$, and the second isomorphism theorem.

We will now mention a few generalizations to modules. In what follows, $R$ will denote a principal ideal ring. This means that $R$ is an integral domain (commutative ring with an identity and no divisors of zero) in which every ideal is principal. By an $R$-module we mean a unitary left $R$-module, and by submodule of an $R$-module we mean a sub- $R$-module. An $R$-module $M$ is called primary if and only if the order ideal of every element of $M$ is generated by a power of the same prime element $p$ of $R$. We shall be content with a generalization to primary modules of our main results for primary groups. We rely heavily on the generalizations of Theorems 1 to 14 in [2].

We make a blanket assertion: All of our lemmas and theorems for primary groups are true for primary modules. Only minor, straightforward modifications of the definitions and proofs are necessary, and these can be easily carried out by imitating all the previous definitions and proofs. When referring to orders of elements in a primary module, we say that $o(x)$ is smaller than $o(y)$ if and only if the generator of the order ideal of $x$ divides the generator of the order ideal of $y$.

In conclusion we state without proof the most worthwhile lemmas and theorems.

Lemma 13. Let $M$ be a primary $R$-module. Let $L, N$ be submodules of $M$ with $L$ containing a basic submodule $B$ of $M$, and $L$ maximal with respect to disjointness from $N$. Then $L$ is pure in $M$.

THEOREM 7. Let $H$ be a high submodule of a primary $R$-module $M$. Then $H$ is pure in $M$.

The solution of Fuchs' question for primary modules is

THEOREM 8. Let $S$ be an infinitely generated submodule of the primary $R$-module $M$ with $R$ countable and $S \cap M^{1}=0$. Then $S$ can be embedded in a pure submodule $K$ of $M$ such that $K \cap M^{1}=0$ and $|K|=|S|$.

The only essential difference between this theorem and Theorem 2 is that the word infinite has been replaced by the words infinitely generated to make $|K|=|S|$ true in all cases. The proof is the same as before. The countability assumption on $R$ makes the proof of [1] p. $78 \mathrm{~N}$ easy.

The author conjectures that all high subgroups of a given primary group are isomorphic, and also wishes to pose the questions: 
For what subgroups $N$ of a primary group $G$ is it true that

(a) all $N$-high subgroups are pure

(b) all $N$-high subgroups are isomorphic

(c) all $N$-high subgroups are endomorphic images of $G$

(d) $G / N$ divisible implies $N$ contains $B$ basic in $G$ ?

\section{REFERENCES}

1. L. Fuchs. Abelian Groups. Publishing House of The Academy of Sciences, Budapest, 1958.

2. Irving Kaplansky. Infinite Abelian Groups. University of Michigan Press, Ann Arbor, 1954.

New Mexico State University and The University of Kansas 


\section{PACIFIC JOURNAL OF MATHEMATICS}

\section{EDITORS}

\author{
RaLPh S. Phillips \\ Stanford University \\ Stanford, California \\ F. H. BRowNELL \\ University of Washington \\ Seattle 5 , Washington
}

A. L. Whiteman

University of Southern California

Los Angeles 7, California

L. J. Paige

University of California

Los Angeles 24, California

\author{
E. F. BECKENBACH \\ T. M. CHERRY
}

\author{
ASSOCIATE EDITORS

$\begin{array}{lll}\text { D. DERRY } & \text { H. L. ROYDEN } & \text { E. G. STRAUS } \\ \text { M. OHTSUKA } & \text { E. SPANIER } & \text { F. WOLF }\end{array}$

\section{SUPPORTING INSTITUTIONS}

\author{
UNIVERSITY OF BRITISH COLUMBIA \\ CALIFORNIA INSTITUTE OF TECHNOLOGY \\ UNIVERSITY OF CALIFORNIA \\ MONTANA STATE UNIVERSITY \\ UNIVERSITY OF NEVADA \\ NEW MEXICO STATE UNIVERSITY \\ OREGON STATE COLLEGE \\ UNIVERSITY OF OREGON \\ OSAKA UNIVERSITY \\ UNIVERSITY OF SOUTHERN CALIFORNIA
}

\author{
STANFORD UNIVERSITY \\ UNIVERSITY OF TOKYO \\ UNIVERSITY OF UTAH \\ WASHINGTON STATE COLLEGE \\ UNIVERSITY OF WASHINGTON \\ AMERICAN MATHEMATICAL SOCIETY \\ CALIFORNIA RESEARCH CORPORATION \\ HUGHES AIRCRAFT COMPANY \\ SPACE TECHNOLOGY LABORATORIES \\ NAVAL ORDNANCE TEST STATION
}

Mathematical papers intended for publication in the Pacific Journal of Mathematics should be typewritten (double spaced), and the author should keep a complete copy. Manuscripts may be sent to any one of the four editors. All other communications to the editors should be addressed to the managing editor, L. J. Paige at the University of California, Los Angeles 24, California.

50 reprints per author of each article are furnished free of charge; additional copies may be obtained at cost in multiples of 50 .

The Pacific Journal of Mathematics is published quarterly, in March, June, September, and December. The price per volume (4 numbers) is $\$ 12.00$; single issues, $\$ 3.50$. Back numbers are available. Special price to individual faculty members of supporting institutions and to individual members of the American Mathematical Society: $\$ 4.00$ per volume; single issues, $\$ 1.25$.

Subscriptions, orders for back numbers, and changes of address should be sent to Pacific Journal of Mathematics, 103 Highland Boulevard, Berkeley 8, California.

Printed at Kokusai Bunken Insatsusha (International Academic Printing Co., Ltd.), No. 6, 2-chome, Fujimi-cho, Chiyoda-ku, Tokyo, Japan.

\section{PUBLISHED BY PACIFIC JOURNAL OF MATHEMATICS, A NON-PROFIT CORPORATION}

The Supporting Institutions listed above contribute to the cost of publication of this Journal, but they are not owners or publishers and have no responsibility for its content or policies.

Reprinted 1966 in the United States of America 


\section{Pacific Journal of Mathematics}

\section{Vol. 11, No. 4}

A. V. Balakrishnan, Prediction theory for Markoff processes . . . . . . . . . . 1171

Dallas O. Banks, Upper bounds for the eigenvalues of some vibrating systems . . . . 1183

A. Białynicki-Birula, On the field of rational functions of algebraic groups ...... 1205

Thomas Andrew Brown, Simple paths on convex polyhedra .............. 1211

L. Carlitz, Some congruences for the Bell polynomials . . . . . . . . . . . . 1215

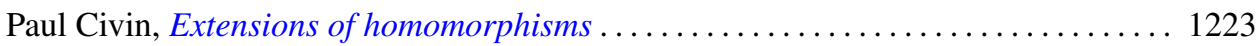

Paul Joseph Cohen and Milton Lees, Asymptotic decay of solutions of differential

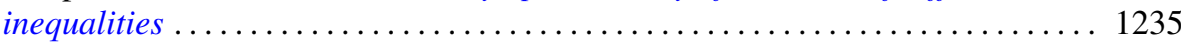

István Fáry, Self-intersection of a sphere on a complex quadric . . . . . . . . . . 1251

Walter Feit and John Griggs Thompson, Groups which have a faithful representation

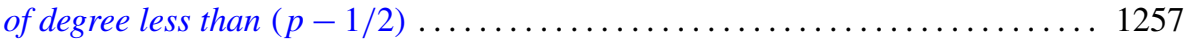

William James Firey, Mean cross-section measures of harmonic means of convex

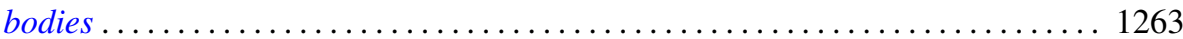

Avner Friedman, The wave equation for differential forms . . . . . . . . . . 1267

Bernard Russel Gelbaum and Jesus Gil De Lamadrid, Bases of tensor products of

Banach spaces ................................... 1281

Ronald Kay Getoor, Infinitely divisible probabilities on the hyperbolic plane . . . . 1287

Basil Gordon, Sequences in groups with distinct partial products . . . . . . . . . . . . 1309

Magnus R. Hestenes, Relative self-adjoint operators in Hilbert space . . . . . . . . . 1315

Fu Cheng Hsiang, On a theorem of Fejér ......................... 1359

John McCormick Irwin and Elbert A. Walker, On N-high subgroups of Abelian

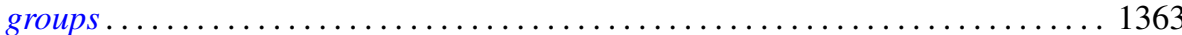

John McCormick Irwin, High subgroups of Abelian torsion groups . . . . . . . . . 1375

R. E. Johnson, Quotient rings of rings with zero singular ideal . . . . . . . . . . . 1385

David G. Kendall and John Leonard Mott, The asymptotic distribution of the time-to-escape for comets strongly bound to the solar system ...

Kurt Kreith, The spectrum of singular self-adjoint elliptic operators ....

Lionello Lombardi, The semicontinuity of the most general integral of the calculus of variations in non-parametric form ................................

Albert W. Marshall and Ingram Olkin, Game theoretic proof that Chebyshev inequalities are sharp

Wallace Smith Martindale, III, Primitive algebras with involution . . William H. Mills, Decomposition of holomorphs ..............

James Donald Monk, On the representation theory for cylindric algebras . . . . . . 1447

Shu-Teh Chen Moy, A note on generalizations of Shannon-McMillan theorem . . . . 1459

Donald Earl Myers, An imbedding space for Schwartz distributions . .

John R. Myhill, Category methods in recursion theory .........

Paul Adrian Nickel, On extremal properties for annular radial and circular slit mappings of bordered Riemann surfaces

Edward Scott O'Keefe, Primal clusters of two-element algebras . .

Nelson Onuchic, Applications of the topological method of Wazewski to certain

problems of asymptotic behavior in ordinary differential equations ...

Peter Perkins, A theorem on regular matrices................

Clinton M. Petty, Centroid surfaces .... 